\title{
An older, more quiescent universe from panchromatic SED fitting of the 3D-HST survey
}

\author{
Joel Leja ${ }^{1}$ D, Benjamin D. Johnson ${ }^{1}$, Charlie Conroy ${ }^{1}$, \\ Pieter van Dokkum ${ }^{2}$, Joshua S. Speagle ${ }^{1}$ and the 3D-HST Team \\ ${ }^{1}$ Harvard-Smithsonian Center for Astrophysics, \\ 60 Garden St. Cambridge, MA 02138, USA \\ ${ }^{2}$ Department of Astronomy, Yale University, New Haven, CT 06511, USA
}

\begin{abstract}
Galaxies are complicated physical systems which obey complex scaling relationships; as a result, properties measured from broadband photometry are often highly correlated, degenerate, or both. Therefore, the accuracy of basic properties like stellar masses and star formation rates (SFRs) depend on the accuracy of many second-order galaxy properties, including star formation histories (SFHs), stellar metallicities, dust properties, and many others. Here, we reassess measurements of galaxy stellar masses and SFRs using a 14-parameter physical model built in the Prospector Bayesian inference framework. We find that galaxies are $\sim 0.2$ dex more massive and have $\sim 0.2$ dex lower star formation rates than classic measurements. These measurements lower the observed cosmic star formation rate density and increase the observed buildup of stellar mass, finally bringing these two metrics into agreement at the factor-of-two level at $0.5<z<2.5$.
\end{abstract}

Keywords. galaxies: evolution, galaxies: formation, galaxies: fundamental parameters

\section{Summary}

This IAU Proceeding is a summary of work published in the Astrophysical Journal under Leja et al. (2019b). Here we apply Prospector, a new code for interpreting galaxy spectral energy distributions (SED) described in Leja et al. (2017), to the photometry from the 3D-HST survey Skelton et al. (2014). The 3D-HST photometric catalog provides observed-frame UV to MIR photometry for $\sim 2 \times 10^{5}$ galaxies over five extragalactic fields totaling $\sim 900 \mathrm{arcmin}^{2}$. Here we use Prospector to fit objects in the redshift range $0.5<z<2.5$ above the observed mass completeness limit for a total of 58,461 objects. We fix the redshift to the photometric redshifts derived with the EAZY code Brammer et al. (2008). We compare the inferred stellar masses and star formation rates from Prospector to classic inference methods for stellar mass and star formation rates, namely the FAST SED-fitting code Kriek et al. (2009) and UV+IR star formation rates Whitaker et al. (2014).

The comparison with the FAST stellar masses can be found in Figure 1. Prospector infers larger stellar masses in low mass galaxies, with the largest systematic offsets around 0.3 dex. This offset decreases with increasing redshifts. A small part of this offset, $\sim 0.05-0.1 \mathrm{dex}$, is due to differing stellar populations codes Leja et al. (2019b). The bulk of the offset is because Prospector infers much older mass-weighted ages than FAST. This is a natural consequence of the nonparametric star formation histories used in Prospector, which remove the bias towards younger ages often found in parametric 


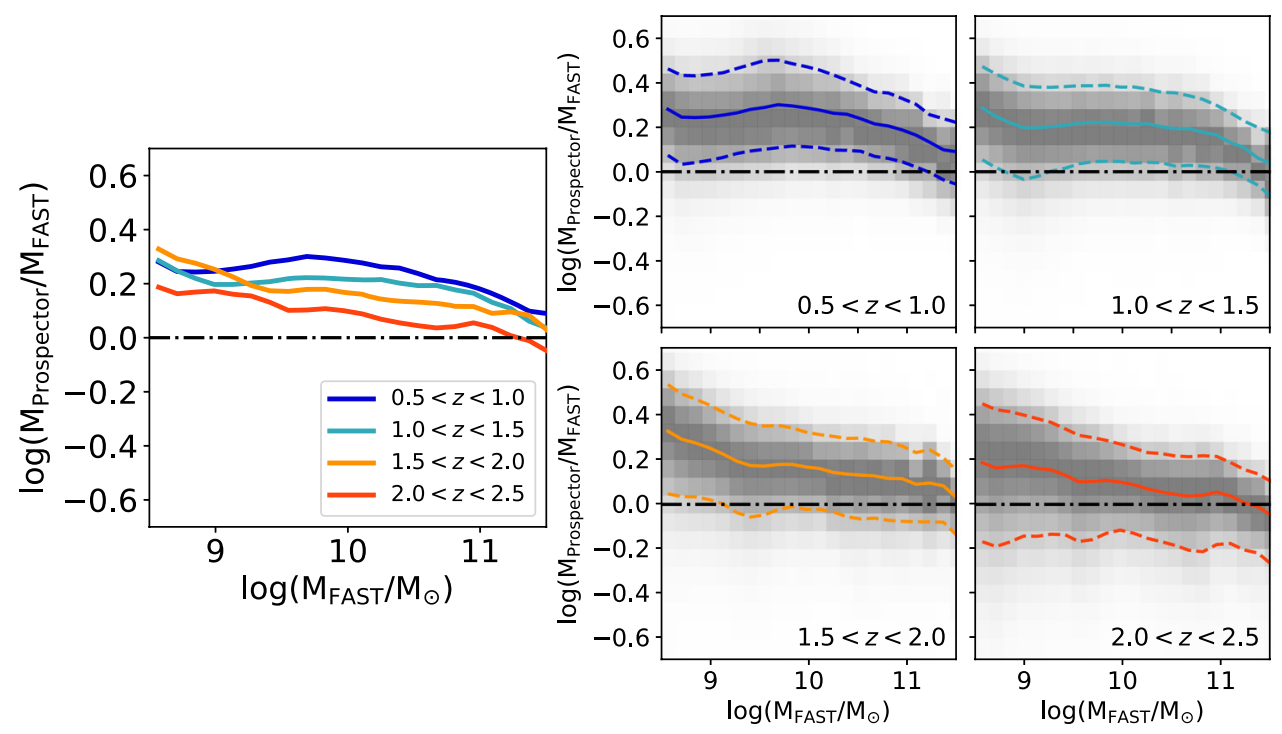

Figure 1. The relative difference between stellar mass derived with Prospector and stellar mass derived with FAST is shown as a function of redshift and stellar mass.
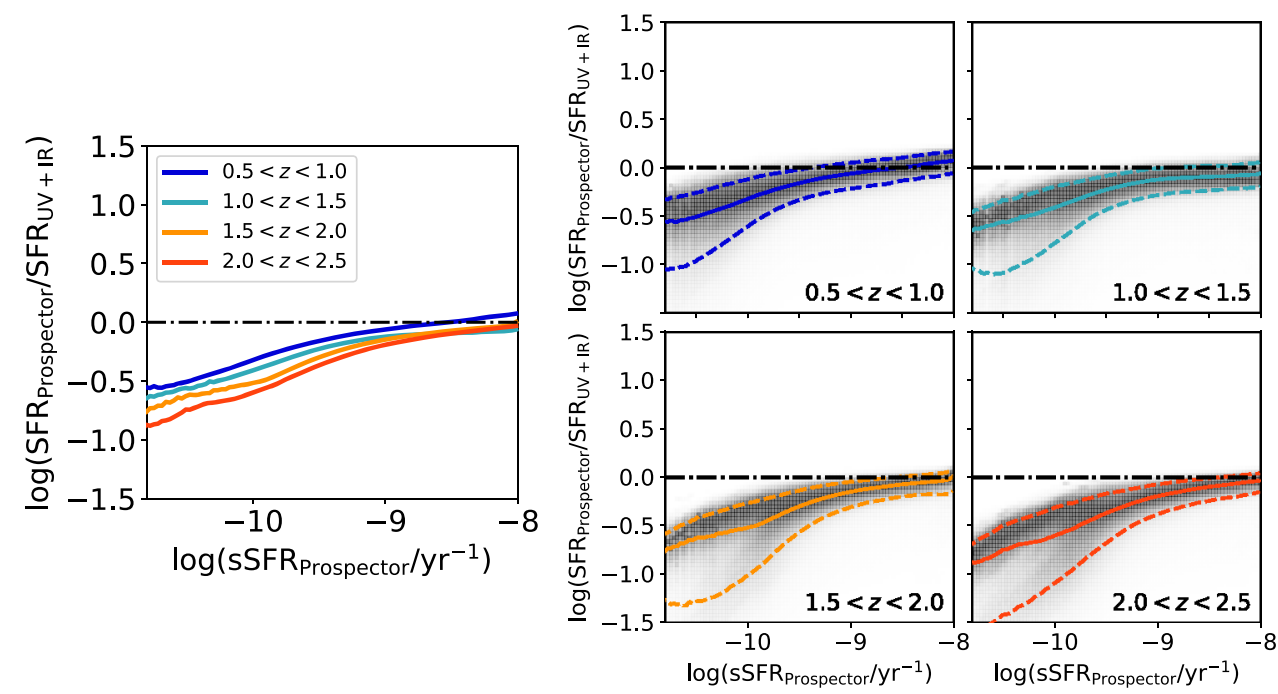

Figure 2. The relative difference between star formation rates derived with Prospector and star formation rates derived with $\mathrm{L}_{U V}$ and $\mathrm{L}_{I R}$ luminosities is shown as a function of redshift and specific star formation rates.

formulae Carnall et al. (2019), Leja et al. (2019a). These older ages create a higher mass-to-light ratio and therefore higher masses.

The comparison with SFRs inferred from UV and IR luminosities can be found in Figure 2. Prospector infers similar star formation rates to UV- and IR-based estimates at high specific star formation rates (sSFRs), but shows an increasing offset towards lower star formation rates at low sSFRs. The median offset is larger at higher redshifts and ranges up to an order-of-magnitude at low sSFRs. Several factors contribute to this 

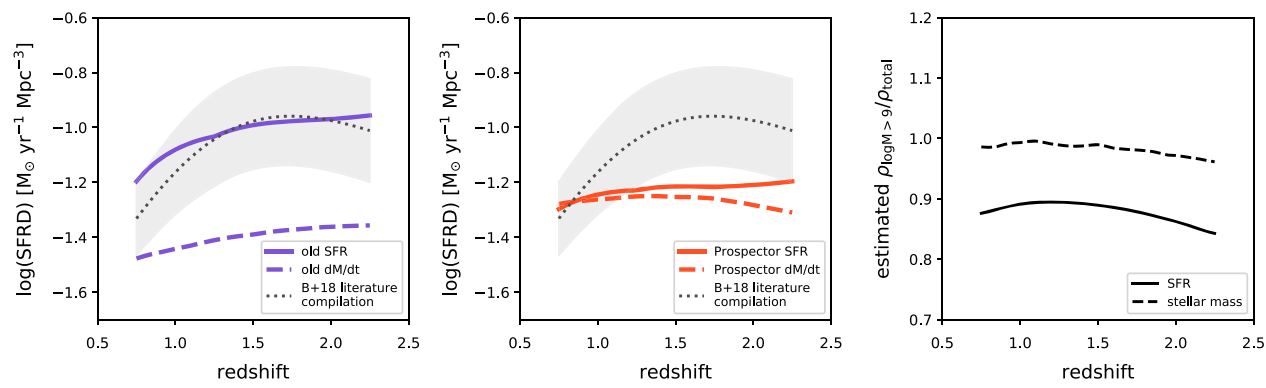

Figure 3. The estimated change in the star formation rate density as inferred independently from the instantaneous star formation rate and from the rate of change of the stellar mass density. The left panel shows the previous estimates while the center panel shows the estimates from Prospector. The right panel shows the estimated incompleteness from the Universe Machine.

effect at a moderate level, including Prospector's accounting for both effects of variable metallicity and active galactic nucleus (AGN) emission in the rest-frame mid-infrared. However, the bulk of the offset comes from the fact that Prospector also models dust heating and UV emission from old stars (i.e. $t>100 \mathrm{Myr}$ ) on an object-by-object basis, while classic estimates based on UV and IR luminosities either neglect or fix this contribution. This explains the increase in the effect at higher redshifts: in the early Universe, the 'old' stars are relatively younger and more luminous. It also explains the increase in the size of the offset at low specific star formation rates, as objects with low sSFRs have relatively more old stars.

We couple these measured systematic offsets with the stellar mass function from Tomczak et al. (2014) in order to estimate the change in the star formation rate density (SFRD). This study estimates the mass function with the FAST SED-fitting code, allowing an apples-to-apples comparison. The SFRD is estimated from two independent sources: the redshift evolution of the mass function and the instantaneous star formation rate of galaxies in some cosmological volume.

For the mass function estimate, we use the smoothed parameterization of the redshift evolution of the stellar mass function from Leja et al. (2015) and the measured $\mathrm{M}_{\text {Prospector }} / \mathrm{M}_{\mathrm{FAST}}$ as a function of $\mathrm{M}_{\mathrm{FAST}}$. We differentiate the mass function with respect to time and integrate over mass in order to measure $\mathrm{d} \rho_{*} / \mathrm{dt}$. Finally we correct for the mass loss rate $R$ in order to turn this into an estimate of $\rho_{\mathrm{SFRD}}$, the instantaneous star formation rate per unit volume. For the estimate directly from the instantaneous star formation rate, we measure $\mathrm{SFR}_{\text {Prospector }}\left(\mathrm{M}_{\mathrm{FAST}}\right)$ and $\mathrm{SFR} \mathrm{UV}_{\mathrm{IR}}\left(\mathrm{M}_{\mathrm{FAST}}\right)$, multiply by the stellar mass function, and integrate in order to estimate $\rho_{\mathrm{SFRD}}$. The incompleteness in both of these quantities due to the lower stellar mass completeness limit of the survey is estimated with the Universe Machine, a semi-empirical model of galaxy formation. This incompleteness is shown in the right-hand panel of Figure 3 and the total SFRDs are corrected for this effect.

The previous inferences of the SFRD are compared with the new inferences from Prospector in Figure 3. The classic inferences are offset from one another at the $\sim 0.3$ dex level, such that there is more observed star formation than stellar mass growth. The Prospector measurements lower the instantaneous star formation rate density by $\sim 0.1-0.2$ dex while increasing the growth of the stellar mass density by $\sim 0.2$ dex. The combination of these effects brings these two independent measurements of the growth of stellar mass into agreement. This suggests that the Prospector values are more reflective of the underlying parameters than the classic estimates, though not conclusively: the behavior below the low-mass limit must be explored to settle this issue, and the effect of 
other cancelling systematic effects such as variation in the high-mass initial mass function may confound the results of this study.

\section{References}

Behroozi, P., Wechsler, R. H., Hearin, A. P., et al. 2019, MNRAS, 488, 3143

Brammer, G. B., van Dokkum, P. G., \& Coppi, P. 2008, ApJ, 686, 1503

Carnall, A. C., Leja, J., Johnson, B. D., et al. 2019, ApJ, 873, 44

Kriek, M., van Dokkum, P. G., Labbé, I., et al. 2009, ApJ, 700, 221

Leja, J., Carnall, A. C., Johnson, B. D., et al. 2019, ApJ, 876, 3

Leja, J., Johnson, B. D., Conroy, C., van Dokkum, P., Speagle, J. S., Brammer, G., Momcheva, I., Skelton, R, Whitaker, \& K. E., Franx, M. 2019, ApJ, 877, 140L

Leja, J., Johnson, B. D., Conroy, C., et al. 2017, ApJ, 837, 170

Leja, J., van Dokkum, P. G., Franx, M., et al. 2015, ApJ, 798, 115

Skelton, R. E., Whitaker, K. E., Momcheva, I. G., et al. 2014, ApJ Supplement, 214, 24

Tomczak, A. R., Quadri, R. F., Tran, K.-V. H., et al. 2014, ApJ, 783, 85

Whitaker, K. E., Franx, M., Leja, J., et al. 2014, ApJ, 795, 104 